Alberta Thy-38-92

CfPA 92-035

UMN-TH-1114/92

November 1992

\title{
Inflation, Neutrino Baryogenesis, and (S)Neutrino-Induced Baryogenesis
}

\author{
Bruce A. Campbell \\ Department of Physics, University of Alberta \\ Edmonton, Alberta, Canada T6G 2J1 \\ Sacha Davidson \\ Center for Particle Astrophysics, University of California \\ Berkeley, California, 94720 USA \\ Keith A. Olive \\ Department of Physics and Astronomy, University of Minnesota \\ Minneapolis, MN 55455, USA
}

\begin{abstract}
We evaluate the constraints that the COBE observations put on baryogenesis in inflationary cosmologies. We consider the supersymmetric version of the proposal of Fukugita and Yanagida, that the baryon asymmetry of the universe is created by nonperturbative electroweak reprocessing of a lepton asymmetry generated in the decay of heavy right handed see-saw (s)neutrinos. We review our recent proposal of a mechanism for baryogenesis via sphaleron reprocessing of a lepton asymmetry generated by (s)neutrino mass effects on flat direction scalar condensate oscillations. Finally we analyze in detail the implementation of these mechanisms in the recently proposed ansatze for fermion mass matrices in supersymmetric, unified, theories.
\end{abstract}

Accepted For Publication In: Nucl.Phys. B 


\section{Introduction}

Baryogenesis [1, 2] is a crucial element in cosmological and particle physics models, and plays a key role in constraining their form. First, the model must be capable of generating a baryon asymmetry. Second, it must be capable of preserving the asymmetry despite the possible dangers of subsequent entropy generation at cosmological phase transitions, or the equilibration of baryon number violating interactions. Models of inflation [3] have always been constrained by baryogenesis. "Sufficient" (for baryogenesis and nucleosynthesis) reheating after inflation is a requirement for any successful inflationary scenario. Non-perturbative standard model electroweak interactions [4] also constrain the structure of possible unification models, because of the danger that a previously established baryon asymmetry may be washed out [4, 5]. In addition, the potential erasure of the baryon asymmetry by sphaleron effects leads to constraints on new interactions violating baryon or lepton number [6, 7, 8, 9, 10, 11, 12].

The fact that baryon number violating interactions in the standard model are relatively unsuppressed [4] at high temperatures, has focussed a great deal of attention on attempts to generate the cosmological baryon asymmetry entirely within the standard model at the relatively low temperature of $0(100 \mathrm{GeV})$ [13, 14, 15]. Recently, more detailed calculations of the effective potential

at finite temperatures [15, 16] have shown however, that the baryon asymmetry is not generated unless the Higgs mass is $m_{H} \lesssim 50 \mathrm{GeV}$, in contradiction with experimental limits [17]. Even in the minimal supersymmetric extension of the standard model, the possibility looks doubtful [18], though non-minimal extensions with a more complicated Higgs sector [19 may turn out to provide viable models of baryogenesis.

Among the simple mechanisms for primordial (before the electroweak scale) baryogenesis are the out of equilibrium decay of a heavy gauge/higgs boson [20, 21], and the decay of a sfermion condensate oscillating along a flat direction in a supersymmetric theory as proposed by Affleck and Dine [22]. Another possibility, proposed by Fukugita and Yanagida 23] (hereafter FY), is the out of equilibrium decay of a heavy neutrino (see also [24]). In this case sphaleron effects are required to transform the lepton asymmetry produced in the decay into a baryon asymmetry. In addition the heavy Majorana neutrino whose decay is responsible for baryogenesis in this mechanism, could induce, via its exchange, a lepton number violating dimension-five effective interaction. If the 
reactions induced by this operator were in thermal equilibrium after the decay of the $N_{R}$, then they would combine with sphaleron interactions to erase the lepton and baryon asymmetry as Fukugita and Yanagida realized [6]. Demanding that the erasure not occur then constrains the induced interaction, and hence the neutrino masses. This double-edged behaviour, that any interactions violating baryon and lepton number, which could in principle cause baryo(lepto)-genesis if out of equilibrium, could equally well erase the BAU if they equilibrate, allows us to derive limits on these interactions from the persistence of a primordial baryon (lepton) asymmetry.

Recently we have proposed an alternate mechanism by which (s)neutrino mass terms in a supersymmetric theory can generated a BAU [25](hereafter CDO). This mechanism also depends on the non-perturbative electroweak reprocessing of a lepton asymmetry. However, in this case, the lepton asymmetry to be reprocessed is generated by the effects of lepton number violating operators, induced by the see-saw (s)neutrino masses, acting on scalar condensate oscillations along flat directions (before supersymmetry breaking) of the supersymmetric theory.

In this paper we wish to explore the connection between inflation and these mechanisms of baryogenesis. In particular we note that the recent COBE observations [26] of the anisotropy of the cosmic microwave background radiation can be related to the reheat temperature after inflation in generic models. We show that inflation may be capable of softening the previously derived constraints on baryon and lepton number violating interactions, inferred from the persistence of a primordial BAU. We then discuss the implications of the derived reheat temperature on models of baryogenesis. We discuss the FY mechanism, and calculate the BAU generated in its extension to supersymmetric models. We discuss the mechanism proposed in CDO, which is a new mechanism for baryogenesis from neutrino Majorana masses, arising in supersymmetric theories. Finally we consider the implementation of both the FY mechanism and the mechanism proposed in CDO via the recently proposed ansatze for (s)neutrino mass matrices, and discuss the relation to neutrino physics and astrophysics. 


\section{Constraints From Inflation}

Consider a generic scalar potential of the form:

$$
V(\eta)=\mu^{4} P(\eta)
$$

where $\eta$ is the scalar field driving inflation, the inflaton; $\mu$ is an as yet unspecified mass parameter, and $P(\eta)$ is a function of $\eta$ which possesses the features necessary for inflation, but contains no small parameter. That is, we expect possible cubic or quartic interactions to be of the form $\lambda_{3} \mu^{4}\left(\eta / M_{P}\right)^{3}$ or $\lambda_{4} \mu^{4}\left(\eta / M_{P}\right)^{4}$, with $\lambda_{3,4} \sim 0(1)$ and $M_{P}$ the Planck mass. Examples of potentials of this form are: "Mexican hat" potentials [27] $V=\mu^{4}\left(1-\eta^{2} / M_{P}^{2}\right)^{2}$ where $\lambda_{4}=1$; potentials used for chaotic inflation 28] $V=\left(\lambda_{4} / 4\right)\left(\mu / M_{P}\right)^{4} \eta^{4}$ or $V=(1 / 2) m^{2} \eta^{2}$ where $m^{2}=2 \mu^{4} / M_{P}{ }^{2}$; Pseudo-Goldstone Boson (PGB) potentials [29 $V=\mu^{4} \cos \theta / f_{\theta}$, which for $f_{\theta} \sim M_{P}$ can be expanded about $\theta=0$, so that $V \simeq \mu^{4}\left(1-\theta^{2} / M_{P}^{2}+\ldots\right)$ exactly as in the expansion of the "Mexican hat" potential above; potentials derived from minimal $N=1$ supergravity superpotentials [30] $F=\mu^{2}\left(1-\eta / M_{P}\right)^{2} M_{P}$;

or potentials derived from no-scale supergravity superpotentials [31] $F=\mu^{2}\left(\eta-\eta^{4} / 4 M_{P}^{3}\right)$. Clearly most of the useful inflationary potentials can be put into the form of Equation (1).

For large scale fluctuations of the type measured by COBE [26], ie. for those which "reenter" the horizon in the matter dominated epoch, density perturbations at horizon crossing are given by 32:

$$
\frac{\delta \rho}{\rho} \simeq \frac{H^{2}}{10 \pi^{3 / 2} \dot{\eta}} \simeq O(100) \frac{\mu^{2}}{M_{P}^{2}}
$$

where the exact coefficient obviously depends on the specific potential under consideration (for chaotic inflation from a quadratic potential, the coefficient is somewhat smaller). The magnitude of the density fluctuations can be related to the observed quadropole 33 moment:

$$
\left\langle a_{2}^{2}\right\rangle=\frac{5}{6} 2 \pi^{2}\left(\frac{\delta \rho}{\rho}\right)^{2}
$$

(we are here assuming that scalar perturbations are dominant [34]). The observed quadropole moment gives [26]:

$$
\left\langle a_{2}^{2}\right\rangle=(4.7 \pm 2) \times 10^{-10}
$$


or

$$
\frac{\delta \rho}{\rho}=(5.4 \pm 1.6) \times 10^{-6}
$$

which in turn fixes the coefficient $\mu$ of the inflaton potential:

$$
\frac{\mu^{2}}{M_{P}^{2}}=f e w \times 10^{-8}
$$

Fixing $\left(\mu^{2} / M_{P}^{2}\right)$ has immediate general consequences for inflation [35]. For example, the Hubble parameter during inflation, $H^{2} \simeq(8 \pi / 3)\left(\mu^{4} / M_{P}^{2}\right)$ so that $H \sim 10^{-7} M_{P}$. The duration of inflation is $\tau \simeq M_{P}^{3} / \mu^{4}$, and the number of e-foldings of expansion is $H \tau \sim 8 \pi\left(M_{P}^{2} / \mu^{2}\right) \sim 10^{9}$. If the inflaton decay rate goes as $\Gamma \sim m_{\eta}^{3} / M_{P}^{2} \sim \mu^{6} / M_{P}^{5}$, the universe recovers at a temperature $T_{R} \sim\left(\Gamma M_{P}\right)^{1 / 2} \sim$ $\mu^{3} / M_{P}^{2} \sim 10^{-11} M_{P} \sim 10^{8} \mathrm{GeV}$. Recall that before COBE all that could be set was an upper limit on $\mu$.

The low reheat temperature and low inflaton mass $m_{\eta} \simeq \mu^{2} / M_{P} \simeq 10^{11} \mathrm{GeV}$ can be quite constraining on models of baryogenesis. In the out of equilibrium decay scenarios, this means that the Higgs bosons in question must have masses $M_{H} \leq m_{\eta} \sim 10^{11} \mathrm{GeV}$. To have Higgs triplets with baryon and lepton number violating interactions this light is problematic for proton stability. Although the Yukawa couplings of these triplet Higgs are related by group theory factors to the Yukawas of the doublet Higgs responsible for the quark and lepton masses, and hence small for the first generation particles appearing in proton decay diagrams, nonetheless, masses for the triplet Higgs of this order would, in many models, cause proton decay at experimentally disallowed rates. The problem goes from troubling to terminal in simple susy-GUTs, where the relatively light Higgs triplets are accompanied by their Higgsino superpartners. Exchange of these particles then induces dimension-five effective interactions, suppressed by only one power of the Higgsino mass. When dressed by external gaugino exchange, one gets a proton decay amplitude down by only one power each of the Higgsino mass, and the chargino (or neutralino or gluino) mass. For gaugino masses of order a $\mathrm{TeV}$ or less, this induces proton decay at a disallowed rate unless the Higgsino mass is of order the susy-GUT unification scale, and hence useless for baryogenesis after inflation. It is possible to avoid the dimension five operators, at the price of complicating the model. For example, in the SU(5) susy-GUT the dimension five operator is induced by the diagram of Figure 1, where the cross denotes the mixing of the Higgs 5 and $\overline{5}$ representations. If one were to duplicate the 
Higgs representation content, so that there were two Higgs 5 and two Higgs $\overline{5}$ representations, where one of each coupled to give the standard masses to the quarks and leptons, and the duplicate "sterile" representation of each did not couple to quarks and leptons, and if one arranged that the mass mixing between the 5 and $\overline{5}$ representations was such that the normal 5 only mixed with the "sterile" $\overline{5}$ and vice versa, then one would have effectively killed the dimension five operator, but at the price of an ad hoc extension of the Higgs sector. Another issue that would need to be addressed in any susy-GUT featuring Higgs triplets below $10^{11} \mathrm{GeV}$ is the effect of these states and their superpartners on the renormalization group equations governing the running of the gauge couplings. While undesired effects on the beta functions for the gauge running might in principle be compensated for by the effects of the addition of other multiplets, the procedure again lacks outside motivation.

Another possibility for generating the baryon asymmetry is the decay of sfermion condensates in a susy-GUT [22. Here the baryon number is generated by the oscillation of scalar fields along a flat direction of the scalar potential [22, 36]. When the dilution of the baryon to entropy ratio due to inflaton decay has been taken into account, the baryon asymmetry is given by [35]:

$$
\frac{n_{B}}{s} \simeq \frac{\delta \phi_{o}^{4} m_{\eta}^{3 / 2}}{M_{X}^{2} M_{P}^{5 / 2} \tilde{m}}
$$

where $\delta$ is a combination of coupling constants parametrizing the CP-violation in the sfermion decay, $\phi_{o}$ is the initial sfermion vev, which is determined by quantum fluctuations during inflation, so that $\phi_{o}{ }^{2} \simeq H^{3} \tau / 4 \pi^{2} \simeq \mu^{2}$ and $\tilde{m} \simeq 10^{-16} M_{P}$ is the supersymmetry breaking scale, and $M_{X} \simeq 10^{-3} M_{P}$ is the unification scale. Then the baryon asymmetry becomes:

$$
\frac{n_{B}}{s} \simeq \frac{\delta \mu^{7}}{M_{X}^{2} M_{P}^{4} \tilde{m}} \simeq 10^{-4} \delta
$$

Of course, with regard to sphalerons, the Affleck-Dine mechanism based on supersymmetric $\mathrm{SU}(5)$ fares no better than the out of equilibrium decay scenario based on $\mathrm{SU}(5)$. The operators involving flat direction fields (LQQQ) with non-vanishing vevs preserve B-L and therefore will have any asymmetry they produce above $T_{c}$ (the electroweak phase transition temperature) erased. Though it may be possible to push down the temperature after the condensate decay, the typical reheat temperature is of order $10^{4} \mathrm{GeV}>T_{c}$. This may not be terribly problematic in unified groups 
beyond SU(5), as they may induce baryon number violating operators which don't conserve B-L,

and involving flat direction fields [37] [38]. What this does suggest, however, is that in the simpler models, we should look for baryogenesis mechanisms that are immune to sphaleron erasure. For these reasons, in the next two sections we turn to baryogenesis mechanisms induced by neutrino Majorana mass terms. We first turn to the FY mechanism, involving the out of equilibrium decay of heavy Majorana right handed neutrinos, and derive its supersymmetric extension. In the succeeding section we turn to the mechanism proposed in CDO, which is only possible in supersymmetric theories, and in which lepton number violating potential terms, induced in the low energy potential (after supersymmetry breaking) from the (s)neutrino Majorana mass superpotential terms, act on scalar condensate oscillations along flat directions of the potential (before supersymmetry breaking). In section five we then examine the implementation of these mechanisms in recently proposed ansatze for neutrino masses in supersymmetric theories, to assess their effectiveness. The advantage of these two mechanisms is that since they are driven by B-L violating Majorana neutrino masses, the non-zero B-L produced is reprocessed to baryons, and sphaleron effects actually cause them to function, rather than posing a danger to the BAU they produce.

\section{$3 \quad$ Neutrino Baryogenesis}

In this section we would like to discuss the possibility of generating the baryon asymmetry by the decay of a massive right handed see-saw (s)neutrino, in a supersymmetric model. In fact, the possibility of generating the baryon asymmetry by the decay of superheavy leptons predates [39] the suggestion of Fukugita and Yanagida [23]. In these earlier works however, the heavy lepton was presumed to decay via baryon number violating GUT interactions, into two or three body final states with non-zero baryon number. For leptons light enough to be produced by thermalization or inflaton decay after inflation, it is difficult to arrange baryon number violating decays without the relatively light fields responsible destabilizing the proton. What is novel and elegant in the FukugitaYanagida proposal, is that the heavy lepton decay directly produces only a lepton asymmetry, and may be arranged to do so with physics as simple as a see-saw neutrino mass matrix. The baryon asymmetry is then subsequently produced by sphaleron reprocessing of the lepton asymmetry, since $L \neq 0$ from the heavy neutrino decay implies $B-L \neq 0$, and the equilibrium condition then results 
in a BAU.

If one adds heavy gauge singlet majorana neutrinos to the Standard Model via the Lagrangian (in two-component notation (uppercase letters for fermions) and in four component notation (lower case))

$$
\begin{array}{r}
i N^{\dagger k} \partial_{\mu} \sigma^{\mu} N^{k}-N^{k} M_{k} N^{k}+\lambda_{k j} H N^{k} L^{j}+\text { h.c. } \\
\quad=\frac{1}{2} \bar{n}^{k}\left(i \not \partial-M_{k}\right) n^{k}+\lambda_{k j} H \bar{n}^{k} P_{L} l^{j}+\text { h.c. }
\end{array}
$$

where the majorana masses $M_{k}$ are large, then below the electroweak phase transition the $\mathrm{SU}(2)$ doublet neutrinos acquire majorana masses of order $\lambda^{2}<H>^{2} / M$ (seesaw). Such light neutrinos could solve the solar neutrino problem or consitute hot dark matter, and, as suggested by Fukugita and Yanagida, the heavy $N^{i}$ could produce a lepton asymmetry when they decay in the early Universe.

To produce an LAU, one needs out-of-equilibrium $L, C$ and $C P$ violating interactions [1]. The majorana mass (or the higgs coupling, depending on whether one assigns lepton number to the $N^{i}$ ) violates $L$, the Universe expansion prevents the decay from being in exact thermal equilibrium, and the phases in the matrix $\lambda$ (combined with the imaginary part of the tree-loop amplitude) provide $C P$ violation. If one defines, as a measure of the $C P$ violation in a decay

$$
\epsilon=\frac{\Gamma-\Gamma_{C P}}{\Gamma+\Gamma_{C P}}
$$

then the lepton asymmetry produced in the out-of-equilibrium decays is $\Delta L \sim 10^{-2} \epsilon$. For the decay $N^{i} \rightarrow H L^{j}$, in which case the CP violation comes from the interference between the tree diagram and figure $2 \mathrm{a}$, this has been calculated to be [23, 24]

$$
\epsilon_{i}=\frac{1}{2 \pi\left(\lambda \lambda^{\dagger}\right)_{i i}} \sum_{j}\left(\operatorname{Im}\left[\left(\lambda \lambda^{\dagger}\right)_{i j}\right]^{2}\right) f\left(M_{j}^{2} / M_{i}^{2}\right)
$$

where

$$
f(x)=\sqrt{x}\left(1-(1+x) \ln \left[\frac{1+x}{x}\right]\right) .
$$

The supersymmetric generalization of (9) would be to add kinetic terms for the gauge singlet superfields (denoted boldface) $\mathbf{N}^{i}$, and superpotential terms

$$
M_{i} \mathbf{N}^{i} \mathbf{N}^{i}+\lambda_{i j} \mathbf{N}^{i} \mathbf{H} \mathbf{L}^{j}+\text { h.c. }
$$


to the Lagrangian for the minimal supersymmetric standard model. We have chosen a basis for the $\mathbf{N}^{i}$ such that the mass matrix is diagonal and real. The $\mathbf{N}^{i}$ in this notation are left-handed, so would be the CP-conjugates of the heavy right-handed neutrinos of the see-saw mass matrix. In component field notation, where we use tildes over scalars (Higgs excepted) to distinguish them from their partner fermions, (13) gives quartic scalar interactions that will not contribute to $C P$ violation at one loop, and

$$
\begin{array}{r}
-\lambda_{i j} M_{i} \tilde{N}^{i *} H \tilde{L}^{j}+\lambda_{i j} \tilde{N}^{i} \tilde{H} L^{j}+\lambda_{i j} H N^{i} L^{j}+\lambda_{i j} \tilde{L}^{j} \tilde{H} N^{i}+\text { h.c. } \\
=-\lambda_{i j} M_{i} \tilde{N}^{i *} H \tilde{L}^{j}+\lambda_{i j} \tilde{N}^{i} \bar{h}^{c} P_{L} l^{j}+\lambda_{i j} H \bar{n}^{i} P_{L} l^{j}+\lambda_{i j} \tilde{L}^{j} \bar{h}^{c} P_{L} n^{i}+\text { h.c. }
\end{array}
$$

The heavy neutrinos $\left(N^{i}\right)$ therefore can decay to $L^{j} H$ or to $\tilde{L}^{j} \tilde{H}$, and the sneutrinos to $\tilde{L}^{j} H$ or $L^{j} \tilde{H}$. The tree level decay rate for each of these processes is

$$
\Gamma_{D}^{i}=\frac{\left(\lambda \lambda^{\dagger}\right)_{i i} M_{i}}{16 \pi}
$$

The one loop diagrams which will contribute to the $\mathrm{CP}$ violating parameter $\epsilon$ in each of these decays are listed in figure 2. Since this is a supersymmetric theory, one might worry that these diagrams cancel; fortunately however, they are contributions to the D-term $\mathbf{N}^{*} \mathbf{H L}$, so superpotential non-renormalization theorems do not apply and a non-zero lepton asymmetry is possible. The CP-violating parameters for the four decays are equal, so the lepton asymmetry due to the out-ofequilibrium decays of the $i$ th generation neutrino and sneutrino is (assuming a maximally out-ofequilibrium decay)

$$
\frac{n_{L}}{n_{\gamma}} \simeq-10^{-2} \frac{1}{2 \pi\left(\lambda \lambda^{\dagger}\right)_{i i}} \sum_{j}\left(\operatorname{Im}\left[\left(\lambda \lambda^{\dagger}\right)_{i j}\right]^{2}\right) g\left(M_{j}^{2} / M_{i}^{2}\right)
$$

where

$$
g(x)=4 \sqrt{x} \ln \frac{1+x}{x}
$$

After sphaleron interactions are included this turns into a baryon asymmetry,

$$
\frac{n_{B}}{n_{\gamma}} \simeq-\frac{28}{79} \frac{n_{L}}{n_{\gamma}}
$$

where $n_{L} / n_{\gamma}$ here is from (16). 
As FY themselves noted [6], integrating the heavy neutrinos (and sneutrinos) out of the Lagrangian (9) ((14) in the supersymmetric case) leads to an effective L violating interaction of the form

$$
\frac{\lambda^{2}}{M} L L H H
$$

which must be out of equilibrium for the baryon (lepton) asymmetry to survive. Thus we must require the rate

$$
\Gamma_{I} \simeq \frac{\zeta(3)}{8 \pi^{3}} \frac{\lambda^{4} T^{3}}{M^{2}}
$$

associated with (19) to be out of equilibrium leading to the constraint [6, 6, 9, 10]

$$
\frac{M}{\lambda^{2}} \gtrsim 1.4 \times 10^{-2} \sqrt{T_{B-L} M_{P}}
$$

The temperature scale in (21) is to be understood as the lowest temperature at which the B$\mathrm{L}$ asymmetry is produced, or $T_{m} \sim 10^{12} \mathrm{GeV}$, the maximum temperature for which sphaleron interactions are in equilibrium. In the FY scenario above, $T_{B-L}$ is just the reheat temperature subsequent to $\mathrm{N}$ decay, $T_{N}$,

$$
T_{B-L}=T_{N} \simeq \sqrt{\frac{\Gamma_{D} M_{P}}{25}}
$$

so that

$$
\frac{M}{\lambda^{2}} \gtrsim \min \left[3 \times 10^{-4} \lambda^{4 / 3} M_{P}, 1.4 \times 10^{-2}\left(T_{m} M_{P}\right)^{1 / 2}\right]
$$

The above analyses was carried out without considering the effects of inflation. In an inflationary model, one must require first that the right-handed neutrino can be produced by inflaton decays, $M<m_{\eta} \sim 10^{11} \mathrm{GeV}$. The lower bound on $\mathrm{M}$ in (23) is also modified. Now,

$$
\frac{M}{\lambda^{2}} \gtrsim \min \left[3 \times 10^{-4} \lambda^{4 / 3} M_{P}, 1.4 \times 10^{-2}\left(\tilde{T}_{R} M_{P}\right)^{1 / 2}\right]
$$

where $\tilde{T}_{R}$ is related to the inflationary "reheat" temperature $T_{R} \sim \mu^{3} / M_{P}^{2} \sim 10^{8} \mathrm{GeV}$; the limit (24) requires equilibrium to be established, and thermalization after inflation occurs only at a temperature $\tilde{T}_{R} \sim \alpha^{2} T_{R}$ 35] where $\alpha$ is a typical gauge coupling constant. Thus $\tilde{T}_{R} \sim 10^{5} \mathrm{GeV}$. Then the lepton asymmetry after $\mathrm{N}$ decay (provided $\lambda$ is not too small) is

$$
\frac{n_{L}}{n_{\gamma}} \simeq \frac{n_{\eta}}{n_{\gamma}} \epsilon \sim\left(\frac{m_{\eta}}{M_{P}}\right)^{1 / 2} \epsilon \sim \frac{\mu}{M_{P}} \epsilon
$$


What is especially important about the above modification due to inflation is that it applies to all of the constraints on dimension five or greater B and L violating operators combined with sphaleron interactions. The constraints on renormalizable operators are all determined by the equilibrium condition at $T=T_{c} \sim O(100) G e V$. Furthermore, the softening of the constraint due to inflation does not depend on supersymmetry. This is in contrast to the important realization by Ibanez and Quevedo [11] of a softening in supersymmetric models due to the presence of additional anomalies. The supersymmetric softening is less severe than the one described here, ie. $T_{\max } \sim 10^{8} \mathrm{GeV}$, due to supersymmetric anomalies.

\section{4 (S)Neutrino-Induced Baryogenesis}

In this section we review the mechanism of baryogenesis recently proposed in CDO [25], arising from the presence of see-saw neutrino masses in supersymmetric theories. We first derive the contributions to the low energy effective potential of a supersymmetric theory, induced after supersymmetry breaking, by the singlet (s)neutrino interactions. We then examine the effect of these interactions on slepton and squark condensates oscillating along "flat directions" (before supersymmetry breaking) of the low-energy supersymmetric standard model. We show that these interactions act on the condensate oscillations to produce a net lepton asymmetry, which nonperturbative electroweak effects partially reprocess into baryons. The mechanism resembles that of Fukugita and Yanagida in that it requires a see-saw neutrino mass, and in that the dynamical mechanism only generates a lepton asymmetry at first, with sphalerons responsible for partially reprocessing that into baryons.

On the other hand, unlike the proposal of Fukugita and Yanagida, the dynamics of this mechanism involves the oscillations of sfermion condensates along susy flat directions, and hence unlike theirs can only occur in supersymmetric extensions of the standard model. Furthermore, in this mechanism the CP violation necessary for the production of the asymmetry may arise spontaneously from the phase of the condensate, and hence is naturally of order unity, whereas in the FukugitaYanagida scenario one needs hard CP violation in the neutrino-Higgs Yukawa couplings. Finally, as this mechanism depends on the effective low-energy interactions induced by the right handed see-saw neutrinos, it can be operative even when the singlet neutrino masses are too large for them to be physically produced in the post-inflationary epoch, whereas in the Fukugita-Yanagida mecha- 
nism they must be copiously produced, thus bounding their mass by the inflaton mass scale, which COBE results give as $\leq 10^{11} \mathrm{GeV}$ for typical inflationary models, as shown above.

In order to have this mechanism of baryogenesis one needs the following elements. First we need to show the existence of flat (before supersymmetry breaking) directions in the potential of our model, including the contributions coming from the extra superpotemtial terms involving the singlet neutrino. The motion, after supersymmetry breaking potentials turn on, of the scalar condensates (squark, slepton, and Higgs) along these directions drives the lepton asymmetry generation. Second, we need to establish the existence of slepton number violating potential interactions, induced after supersymmetry breaking by the neutrino mass see-saw superpotential terms, which pick up a nonzero contribution along the flat direction, and which act during the course of the scalar oscillations to build up a net slepton density. Third, we must follow the evolution of the condensate to calculate the lepton asymmetry produced, and its subsequent dilution by inflaton decay, to get the final lepton asymmetry for sphaleron reprocessing.

To demonstrate the mechanism, we need to exhibit flat directions in the supersymmetric standard model, extended to include neutrino see-saw masses which arise from the superpotential (13), and renormalizable $N$-field superpotential terms $k_{1}^{i} \mathbf{N}^{i} \mathbf{H}_{1} \mathbf{H}_{2}+k_{2}^{i j k} \mathbf{N}^{i} \mathbf{N}^{j} \mathbf{N}^{k}$. Note that the singlet neutrino interactions in this section therefore differ from those in the previous one; the superpotential terms involving $\mathbf{N}^{\mathbf{i}}$ are

$$
M_{i} \mathbf{N}^{i} \mathbf{N}^{i}+\lambda_{i j} \mathbf{N}^{i} \mathbf{H L}^{j}+k_{1}^{i} \mathbf{N}^{i} \mathbf{H}_{1} \mathbf{H}_{2}+k_{2}^{i j k} \mathbf{N}^{i} \mathbf{N}^{j} \mathbf{N}^{k}+h . c .
$$

The potential from these and the usual standard model F-terms (plus the $\mathrm{SU}(3) \mathrm{xSU}(2) \mathrm{xU}(1) \mathrm{D}$ terms) has, among others, the following flat direction (which is a generation permuted version of one appearing in reference [38]); it depends on three arbitrary complex parameters $a, v, c$, and four phases $\alpha, \beta, \phi$, and $\gamma$. We work in a generation basis in which the $g_{e}^{i j}$ and the $g_{d}^{i j}$ have been diagonalized; the quark indices denote quark colour.

$$
\begin{array}{ccc}
\tilde{t}_{3}^{c}=a & \tilde{t}^{1}=v & \tilde{\nu}_{e}=e^{i \gamma} c \\
\tilde{b}_{3}^{c}=c & \tilde{s}_{2}^{c}=e^{i \alpha} \sqrt{|a|^{2}+|c|^{2}} & \\
\tilde{\mu}^{-}=e^{i \beta} \sqrt{|v|^{2}+|c|^{2}} & \tilde{d}_{1}^{c}=e^{i \phi} \sqrt{|a|^{2}+|v|^{2}+|c|^{2}}
\end{array}
$$

A vev along this particular flat direction produces a non-zero vev for the effective scalar operator

$$
\left\langle\tilde{\mu}^{-} \tilde{\nu}_{e} \tilde{b}_{3}^{c}\left(\tilde{t}_{3}^{c}\right)^{*}\right\rangle=e^{i(\beta+\gamma)} a^{*} c^{2} \sqrt{|v|^{2}+|c|^{2}}
$$


which violates lepton number by two units.

After supersymmetry breaking, this scalar operator will be induced by the singlet (s)neutrino interactions via the diagram of Figure 3. In the diagram the insertions on the $\tilde{N}$ line and vertex are the supersymmetry breaking scalar mass and interaction A-terms $\left(O\left(m_{\delta}\right)\right)$. The resulting potential term coupling is of order $V=\lambda \phi \phi \phi \phi^{*}$, where $\phi \phi \phi \phi^{*}$ corresponds to the quartic scalar operator of equation (28) and

$$
\lambda \simeq \frac{g_{\nu}^{e} g_{\nu}^{\mu} g_{b} g_{t}}{(4 \pi)^{3}} \frac{m_{\delta}^{2} M^{2}}{\left(M^{2}+g^{2} \phi_{o}^{2}\right)^{2}}
$$

where $g_{\nu}^{e}$ and $g_{\nu}^{\mu}$ are the (experimentally undetermined) neutrino see-saw Dirac mass Yukawas, and $\mathrm{M}$ is the scale of the large singlet $\mathbf{N}$ Majorana mass term (We assume $k_{1}, k_{2} \sim 1$ ). This estimate for the scale of the induced quartic scalar coupling is supported by the general arguments of [38] for operators of this form.

The calculation of the lepton (and baryon) asymmetry produced by the sfermion condensate was undertaken in CDO [25]. If we denote the expectation values, after inflation, of scalars parametrizing the flat directions as $\phi_{o}=\langle o|\phi| o\rangle$, producing $V_{o}=\langle o|V| o\rangle$, then we can then write the net lepton number per scalar particle associated with the oscillations of $\phi$ as

$$
L \sim \frac{I m V_{o}}{m_{\delta}{ }^{2} \phi_{o}{ }^{2}} \sim \frac{\theta \lambda \phi_{o}{ }^{4}}{m_{\delta}{ }^{2} \phi_{o}{ }^{2}} \sim O\left(10^{-5}\right) \theta g_{\nu}^{e} g_{\nu}^{\mu} \frac{\phi_{o}{ }^{2} M^{2}}{\left(M^{2}+g^{2} \phi_{o}^{2}\right)^{2}}
$$

where $\theta \sim 1$ is the degree of CP violation in (28) and we have assumed that $g_{t} \sim 1$ and $g_{b} \sim O\left(10^{-2}\right)$. The net lepton number density ( $\sim$ the net baryon density after sphaleron reprocessing) is then given by

$$
n_{B} \sim n_{L} \sim \operatorname{Lm}_{\delta} \phi_{o}^{2}\left(R_{\phi} / R\right)^{3}
$$

where $\mathrm{R}$ is the cosmological scale factor and $R_{\phi}$ is the value of the scale factor when the sfermion oscillations begin.

To evaluate the BAU produced after inflation by this mechanism, we must recall that the initial value of $\phi_{o}$ is determined by quantum fluctuations during inflation, and that the the asymmetry is diluted by inflaton decays. The initial sfermion expectation value is $\phi_{o}{ }^{2} \simeq H^{3} \tau / 4 \pi$ where the Hubble parameter $H \simeq \mu^{2} / M_{P}$ and the duration of inflation is $\tau \simeq M_{P}^{3} / \mu^{4}$ so that $\phi_{o} \simeq \mu$, where our discussion above of COBE indicates $\mu^{2} \simeq$ few $\times 10^{-8} M_{P}^{2}$. The final baryon asymmetry can 
then be found from [35] to be

$$
\frac{n_{B}}{n_{\gamma}} \simeq O\left(10^{-5}\right) \frac{\theta g_{\nu}^{e} g_{\nu}^{\mu} \phi_{o}^{4} M^{2} m_{\eta}^{3 / 2}}{\left(M^{2}+g^{2} \phi_{o}^{2}\right)^{2} M_{P}^{5 / 2} m_{\delta}} \simeq O\left(10^{-2}\right) \frac{\theta g_{\nu}^{e} g_{\nu}^{\mu} \mu^{3} M^{2}}{M_{P}{ }^{4} m_{\delta}}
$$

where $m_{\eta} \simeq \mu^{2} / M_{P}$ is the inflaton mass, and we have assumed $M \lesssim g \phi_{o}$ and $g^{4} \sim 10^{-3}$ in the denominator. This gives a value of $\simeq 10^{-10}$ for $\theta g_{\nu}^{e} g_{\nu}^{\mu} M^{2} / M_{P}^{2} \simeq 10^{-13}$, which we will evaluate below for realistic models.

In summary, this mechanism for baryogenesis in supersymmetric extensions of the standard model, does not involve (super)GUT interactions. It depends on non-perturbative electroweak reprocessing of a lepton asymmetry, which is in turn generated by the effects of lepton number violating induced operators, acting on scalar condensate oscillations along flat directions of the standard model. In our realization of the mechanism we have shown that lepton number violating operators of this type can be induced, after supersymmetry breaking, by singlet neutrino interactions that include see-saw neutrino masses. In principle, any other superpotential interaction inducing violation of either baryon or lepton number has the potential to induce baryogenesis via sfermion condensate dynamics, coupled with sphaleron reprocessing, in a manner similar to that of the example we have presented. In the next section we consider the concrete implementation of this scenario utilizing neutrino Majorana masses as discussed above, in the context of recently proposed ansatze for (s)neutrino mass matrices in supersymmetric theories. We compare the BAU produced via this mechanism to that from the Fukugita-Yanagida mechanism, in the same class of theories.

\section{5 (S)Neutrino Masses}

In this section we wish to address the question of whether the mechanisms discussed in the last two sections provide sufficient baryogenesis in the context of realistic models of neutrino masses. By realistic models, we understand that the resulting framework should be theoretically well motivated, predictive, and giving experimentally succesful predictions for fermion masses. In the modern context, this usually means finding a ground state solution to heterotic superstring theory, that is well motivated and phenomenologically viable. This is, of course, an exceptionally ambitious undertaking, as such a solution would encompass all of particle physics, and despite vigorous efforts 
at expanding the space of known string constructions, at present no solution is known that is completely viable phenomenologically. In view of this, we defer the task of such a construction, and adopt the strategy of working with predictive ansatze for the fermion masses that have been incorporated in grand unified models [40], and that when renormalization group extrapolated from unification scales to accessible scales in supersymmetric theories give experimentally interesting predictions 41]. In general we shall be agnostic with regard to the origin of our fermion mass matrices. As they were first proposed in the context of an $\mathrm{SO}(10)$ grand unified theory [40, with its accompaning GUT gauge and (rather extensive) Higgs sectors, we will consider the physical implications of such an origin for them. However, we will also consider how the physics would appear if we only retain the pattern of the mass matrices as a phenomenological ansatze, and introduce no gauge or Higgs representations or interactions beyond those of the supersymmetric standard model. We expect that these two, rather extreme, approaches will bracket the range of models one might reasonably expect. In particular, string constructions might be expected to lie somewhere between the extremes, as in general both the gauge and Higgs sectors are less populous in string models than GUTs, (but more populous than the susy standard model) due to the possibility of gauge symmetry breaking by "twisting" in string models.

The specific pattern or "texture" of fermion masses that we will assume for our considerations is that proposed by Harvey, Ramond and Reiss in an SO(10) GUT [40](hereafter HRR). Their fermion mass texture includes an up quark mass matrix incorporating the Fritzsch ansatze [42], and down and charged-lepton mass matrices incorporating the Georgi-Jarlskog ansatze [43]; the resulting pattern of quark masses yields Oakes-type relations for the mixing angles. As it was implemented in an SO(10) model, where neutrinos of both chiralities are incorporated in the 16 representations of fermions, it also predicts a see-saw pattern for the neutrino masses [44]. In particular the $\Delta I_{W}=1 / 2$ Dirac mass terms for the neutrinos are related to the up quark mass matrices, and $\Delta I_{W}=0$ Majorana masses for right handed neutrinos are constrained by the $\mathrm{SO}(10)$ Higgs representation content and the discrete symmetries used to enforce the texture. In order to be able to enforce the fermion mass texture by discrete symmetries HRR utilized a rather extensive Higgs sector representation content (we here give the formulation of the model that directly extends to the supersymmetric version, and which is equivalent to the form in their original paper), including a complex 10 and three separate $1 \overline{2} 6$ in the (superpotential) Yukawas, as well as a 54 for GUT 
symmetry breaking. The superpotential Yukawas responsible for masses for the standard model fermion 16 s are:

$$
L_{Y}=\left(A 16_{1} \cdot 16_{2}+B 16_{3} \cdot 16_{3}\right) \cdot 1 \overline{2}_{1}+\left(a 16_{1} \cdot 16_{2}+b 16_{3} \cdot 16_{3}\right) \cdot 10+\left(c 16_{2} \cdot 16_{2}\right) \cdot 1 \overline{2}_{2}+\left(d 16_{2} \cdot 16_{3}\right) \cdot 1 \overline{2} 6_{3}
$$

The discrete symmetry responsible for the HRR texture of fermion masses is spontaneously broken at the unification scale by the Higgs vevs, and hence as one extrapolates to lower energies by the renormalization group equations, the renormalization group mixing will not preserve the texture, modifying the form of the mass matrices at lower energies. Recently, in a series of interesting papers, Dimopoulos, Hall and Raby [1](DHR)have shown that if one uses the renormalization group equations appropriate for the supersymmetric extension of the standard model, then starting with a unification scale texture of the HRR form one generates at the electroweak scale a pattern of quark and lepton masses that is in agreement with present experiment, and which gives testable predictions for future experiments. They have also analyzed the extrapolation of neutrino masses for a specific case of the HRR neutrino mass texture, when only one vev contributes to a given entry of the neutrino mass matrix, and have analyzed the resulting predictions for solar and terrestrial neutrino physics. We will find interesting features of the DHR restriction on neutrino masses of the HRR form, when we discuss the FY mechanism.

Let us now examine neutrino mass matrices of the HRR form. As there are two neutral Weyl spinors per family of 16 , there are a total of 6 neutral lepton spinors. Of these three are the standard $S U(2)_{W}$ doublet neutrinos of the standard model, and three are singlets. The structure of the 6x6 neutral fermion mass matrix is thus:

$$
M=\left(\begin{array}{cc}
M^{(1)} & M^{(1 / 2)} \\
M^{(1 / 2)^{T}} & M^{(0)}
\end{array}\right)
$$

where the $M^{\Delta I_{W}}$ are $3 \times 3$ generation matrices, whose superscript represents their $\Delta I_{W}$ value. Following HRR we assume that there are no $\Delta I_{W}=1$ Higgs expectation values, so that $M^{(1)}=0$. The isosinglet mass $M^{(0)}$ is given by the vev of the $1 \overline{2}_{1}$ along the $\Delta I_{W}=0$ direction.

$$
M^{(0)}=\left(\begin{array}{ccc}
0 & \bar{A} & 0 \\
\bar{A} & 0 & 0 \\
0 & 0 & \bar{B}
\end{array}\right)
$$


where $\bar{A}=A k e^{i \zeta}$, and $\bar{B}=B k e^{i \zeta}$, with $k e^{i \zeta}$ the $\Delta I_{W}=0$ vev of $1 \overline{2}_{1}$.

Also following HRR, we take (with some minor amendments)

$$
M^{(1 / 2)}=\left(\begin{array}{ccc}
0 & \bar{U} & 0 \\
\bar{U} & 0 & -3 \bar{Q} \\
0 & -3 \bar{Q} & \bar{W}
\end{array}\right)
$$

where $\bar{U}=a p e^{i \delta}-3 A t e^{i \sigma}, \bar{W}=b p e^{i \delta}-3 B t e^{i \sigma}$, and $\bar{Q}=d q e^{i \mu}$ where $a, A, b, B$, and $d$, are couplings from the superpotential (33), and $t e^{i \sigma}$ is the $\Delta I_{W}=(1 / 2)$ vev of the $1 \overline{26}{ }_{1}, q e^{i \mu}$ is the $\Delta I_{W}=(1 / 2)$ vev of the $1 \overline{2}_{3}$, and $p e^{i \delta}$ is the $\Delta I_{W}=(1 / 2)$ vev of the complex 10 (appearing in the Higgs doublet with the same weak hypercharge). Since in the low-energy effective supersymmetric theory, we expect these mass terms to arise from the Yukawa coupling to a single Higgs doublet of the required weak hypercharge (which evidently must be a linear combination of the complex 10, $1 \overline{2}_{1}$, and $1 \overline{2}_{3}$, in the ratios of $p, t$, and $q$ ), we may write the mass matrix in terms of the vev of that Higgs in the form:

$$
M^{(1 / 2)}=\left(\begin{array}{ccc}
0 & -3 u & 0 \\
-3 u & 0 & -3 x \\
0 & -3 x & -3 w
\end{array}\right) \frac{v}{\sqrt{2}} \sin \beta
$$

where $u, x$, and $w$ are chosen so that the mass matrix (37) agrees with (36).

The heavy right-handed neutrino mass matrix is made real and diagonal by a redefinition of the right handed neutrino fields: $N=U N^{\prime}$, where

$$
U=\frac{1}{\sqrt{2}}\left(\begin{array}{ccc}
i e^{i \theta} & e^{i \theta} & 0 \\
-i e^{i \theta} & e^{i \theta} & 0 \\
0 & 0 & \sqrt{2} e^{i \phi}
\end{array}\right)
$$

with $\bar{A}=|A k| e^{-2 i \theta}$, and $\bar{B}=|B k| e^{-2 i \phi}$, yielding

$$
N^{T} M^{(0)} N=N^{\prime T} U^{T} M^{(0)} U N^{\prime}=N^{\prime T} D N^{\prime}
$$

where $D=U^{T} M^{(0)} U$ and is given by

$$
D=\left(\begin{array}{ccc}
|A| & 0 & 0 \\
0 & |A| & 0 \\
0 & 0 & |B|
\end{array}\right)
$$


To determine whether or not there are any useful CP-violating phases to generate $L \neq 0$ from (16), we consider the $\nu_{L}-N^{\prime}$ Higgs Yukawas from (37)

$$
N^{T} M^{(1 / 2)} \nu_{L}=N^{\prime T} U^{T} M^{(1 / 2)} \nu_{L}
$$

and the Yukawa couplings $\lambda_{i j}$ can be simply read off

$$
\lambda_{i j}=\frac{1}{\sqrt{2}}\left(\begin{array}{ccc}
3 i u e^{i \theta} & -3 i u e^{i \theta} & 3 i x e^{i \theta} \\
-3 u e^{i \theta} & -3 u e^{i \theta} & -3 x e^{i \theta} \\
0 & -3 \sqrt{2} x e^{i \phi} & -3 \sqrt{2} w e^{i \phi}
\end{array}\right)
$$

By direct computation we find,

$$
\begin{aligned}
\epsilon_{1} & =\frac{1}{2 \pi} \frac{9 m(z)}{\left(2 u^{*} u+x^{*} x\right)} \operatorname{Im}\left[\left(-i u^{*} x+i x^{*} w\right)^{2} e^{2 i(\phi-\theta)}\right] \\
\epsilon_{2} & =\frac{1}{2 \pi} \frac{9 m(z)}{\left(2 u^{*} u+x^{*} x\right)} \operatorname{Im}\left[\left(u^{*} x+x^{*} w\right)^{2} e^{2 i(\phi-\theta)}\right] \\
\epsilon_{3} & =\frac{1}{2 \pi} \frac{18 m(1 / z)}{\left(w^{*} w+x^{*} x\right)} \operatorname{Im}\left[x^{*} u w^{*} x e^{2 i(\theta-\phi)}\right]
\end{aligned}
$$

where $z \equiv\left(M_{3}^{2} / M_{1}^{2}\right)$ and $m(z)$ is $f(z)$ or $g(z)$ (equations (12) and (17) respectively) for the nonsupersymmetric or supersymmetric case. As one can see there are non-vanishing phases for CPviolation.

We also look at the more restrictive ansatz of DHR, where only the vev from a single Higgs multiplet contributes to an element of the mass matrix. In this case $u$ and $w$ are proportional to $\bar{A}$ and $\bar{B}$ respectively, and carry the same phases. So in this case we have:

$$
\begin{gathered}
\epsilon_{1}=\frac{1}{2 \pi} \frac{9 m(z)}{\left(2 u^{*} u+x^{*} x\right)} \operatorname{Im}\left[-|u|^{2} x^{2} e^{2 i(\phi+\theta)}-|w|^{2} x^{* 2} e^{2 i(\phi+\theta)}\right] \\
\epsilon_{2}=-\epsilon_{1} \\
\epsilon_{3}=0
\end{gathered}
$$

Note that even in this special case the $\mathrm{CP}$ violating asymmetry is non-vanishing, and depends on phases which are not fixed by low energy measurements.

In this case one also notes that the asymmetry is equal and opposite for the two, degenerate, lighter right-handed neutrino mass eigenstates. This means that if after inflation one reheated 
above the mass of these states, populating them thermally (hence equally, since they are degenerate), then their resulting decays would produce cancelling asymmetries, to the order in which we have calculated. The cancellation would presumably be vitiated by differences in the asymmetries generated by interferences at two loops. On the other hand, in the case where the two mass eigenstates are populated by inflaton decay, there is no reason to expect equal numbers of them to be produced. Even if the inflaton decays to $\mathrm{N}$ states in a way that is completely flavour symmetric in the chiral basis (unprimed), after the rotation to the mass eigenbasis there will be in general a difference in the numbers produced (unless in the initial chiral basis the flavour dependence in the decay mechanism was fine-tuned to be diagonal). In general, then, the resulting asymmetry will depend on the fraction of the inflaton decays to the first $\mathrm{N}$ mass eigenstate, minus the fraction of decays to the second, degenerate, $\mathrm{N}$ mass eigenstate. Let us denote this difference of fractions by $y$.

To get an idea of how large an asymmetry might be produced with this mechanism with these couplings, let us assume that the undetermined phases appearing in $\epsilon_{1}$ are generically of order unity. DHR have done fits to the solar neutrino data (and extracted the corresponding laboratory predictions) with a neutrino mass ansatze of this type (their "case 1" for which they can fit the combined data). In particular the $\Delta I_{W}=(1 / 2)$ mass terms are related to the charge $2 / 3$ quark masses, and the parameters extracted from their fits to quark masses. The quark mass fits indicate $w>>x>>u$ meaning $z=|w|^{2} /|u|^{2}>>1$ and with $m(z) \sim 1 / \sqrt{z}$ for large $\mathrm{z}$, we find for maximal phases that

$$
\epsilon_{1} \sim \text { few }|u||w|
$$

the quark mass fits indicate: $|w| \simeq 1$, and $|u| \simeq .5 \times 10^{-3}$, giving $\epsilon_{1} \sim 10^{-3}$ for maximal phases. The MSW fit to the solar neutrino data indicates a $N^{\prime}{ }_{1}$ mass of a few times $10^{10} \mathrm{GeV}$. This is consistent with $N^{\prime}{ }_{1}$ production in inflaton decay, given our estimate of the inflaton mass from COBE of order $10^{11} \mathrm{GeV}$. With our estimates for the inflaton mass and reheat temperature we then find:

$$
\frac{n_{B-\bar{B}}}{n_{\gamma}} \sim 10^{-4} y \epsilon
$$

which for maximal phases gives sufficient baryogenesis for $y$ as small as $10^{-4}$. So with parameters determined from predictive ansatze for neutrino masses, and fit to the solar neutrino MSW oscillations, one can have sufficient baryogenesis by the Fukugita-Yanagida mechanism (see also [45]). 
Turning now to the scenario proposed in CDO for (s)neutrino mass induced generation of a BAU, we find that there may be significant differences in the efficacy of the mechanism, depending on how the mass matrix texture has been implemented in the (unified) theory. The issues involve the availability of the couplings required to induce the lepton number violating scalar condensate potential, and finding flat directions along which one can have scalar condensate oscillations.

Since the efficiency of flat direction oscillation mechanisms for baryogenesis in inflationary cosmologies depends in part on the fact that inflationary fluctuations will in general drive the scalars producing the operator vev, to vevs $\phi_{o} \simeq \mu$ of the same order as the unification scale, we must demand F-flatness including all superpotential couplings to GUT sector fields. As one includes more Higgs representations, and larger Higgs representations, which couple to the matter 16s, one increases the number of F-terms whose vanishing is required for the existence of the desired flat directions. As one assembles generations of matter multiplets in single irreducible representations of the gauge group, one reduces the number of independent relative generation rotations one might perform to avoid F-terms. So the more extensive the GUT sector structure, the more difficult it becomes to find useful flat directions.

We see this clearly in the class of models on which we are focusing. If we were to consider the Yukawa coupling pattern with HRR texture as just a pattern of couplings of the standard model superfields, and restrict ourselves to only the standard model multiplets, then we would have (among others) the flat direction which we discussed in the previous section. Assuming the relation between neutrino Dirac mass Yukawas and up quark mass Yukawas implicit in this pattern (though now without any GUT sector), and setting the scale of right handed neutrino masses of order $10^{11} \mathrm{GeV}$, as suggested by the MSW solution of the solar neutrino puzzle 41, and observable sector supersymmetry breaking scalar masses of order $100 \mathrm{GeV}$, we simply plug into equation (32) to determine the resulting BAU:

$$
\frac{n_{B}}{n_{\gamma}} \simeq O\left(10^{-2}\right) \frac{\theta g_{\nu}^{e} g_{\nu}^{\mu} \mu^{3} M^{2}}{M_{P}{ }^{4} m_{\delta}} \sim O\left(10^{-19}\right) \theta
$$

which is clearly insufficient. The origin of the numerical deficiency is the identification $M \lesssim$ $10^{11} \mathrm{GeV}$, which we made in order to match our ansatze to the MSW solution of the solar neutrino problem. Should we relax the requirement of MSW resolution of the neutrino puzzle then we can 
easily raise $M$ sufficiently to provide baryogenesis within our ansatze, with the $\nu_{L}$ see-sawing to unobservably light masses.

Consider now the full SO(10)theory, as elaborated by HRR. Its superpotential includes the terms shown in equation (33), responsible for the generation of standard model fermion masses, plus an extensive set of Higgs self couplings (not shown) which are required to generate the neccessary symmetry breaking vevs. The first thing that we note is that since the "right-handed" neutrino now appears in a 16 of $\mathrm{SO}(10)$, the $\mathbf{N}^{\mathbf{3}}$ and $\mathbf{N H}_{\mathbf{1}} \mathbf{H}_{\mathbf{2}}$ superpotential terms previously used in generating the lepton number violating condensate potential terms, are now forbidden by gauge invariance. However, with the introduction of an $\mathrm{SO}(10)$ singlet coupling to itself and the Higgs 10, one has interactions that induce (at four loops!) the required operator. For flat directions, we do not consider ones involving Higgs vevs, as they would be required to satisfy F-flatness conditions associated with the GUT Higgs self couplings which are very model dependent and which we have not listed, and also as they risk vitiating the generation trick on which we will be forced to rely on below, to avoid F-terms when giving vevs to chiral scalars in the 16s. That we will be forced to rely on a generation trick when introducing vevs in the $16 \mathrm{~s}$ can be seen as follows. In the superpotential Yukawas there are couplings $16 \cdot 16 \cdot 1 \overline{2} 6$ for each of the $1 \overline{2} 6 \mathrm{~s}$. Let us consider the coupling of a $1 \overline{2} 6$ to a single (generation of) 16. Now consider a particular component of the 16 to which we wish to give a vev. By a choice of basis for the subgroup decomposition we may choose this state in the 16 to be the singlet in the $\mathrm{SO}(10) \supset \mathrm{SU}(5) \mathrm{xU}(1)$ decomposition $16=1+\overline{5}+10$. Under $\mathrm{SU}(5) \mathrm{xU}(1)$ decomposition the $1 \overline{2} 6$ also contains a singlet. Furthermore, by inspection of the decomposition of the tensor product $16 \cdot 16 \cdot 1 \overline{2} 6$ under $\mathrm{SU}(5) \mathrm{xU}(1)$ it is clear that the singlet in the $1 \overline{2} 6$ is that appearing (with the same choice of subalgebra basis) in the product of singlets in the 16s. Since by hypothesis this basis was chosen such that the singlet of the 16 was the component receiving a vev, this means that the F-term for the singlet component of the $1 \overline{2} 6$ is nonvanishing, as in the product it is only coupled to the nonvanishing singlets in the 16. In order to evade this argument, we require several generations of 16, such that some linear combination of them has vanishing (generation) diagonal coupling to itself. Inspecting the pattern of superpotential Yukawas in the HRR GUT [equation (33)] we observe that this is exactly what occurs for the $16_{1}$; the discrete symmetry used to enforce the HRR texture has been precisely arrange to prevent its self coupling. So provided vevs in the other $16 \mathrm{~s}$, and the Higgs representations, vanish, any pattern of vevs in the $16_{1}$ will 
be F-flat. It only remains to satisfy the D-flatness condition. As shown in [22], we only have to consider D-terms associated with generators unbroken at the GUT scale. Since the standard model gauge group has 12 generators, there are 12 non-trivial quadratic equations that must be satisfied for D-flatness. We have 16 complex scalars in our generation; the $\mathrm{SU}(5) \mathrm{xU}(1)$ singlet is singlet under the standard model generators (in the basis where the standard model group is embedded in the $\mathrm{SU}(5)$ ) and hence the vev for the singlet receives no D-term contributions. For the remaining 15 chiral scalars we have 12 conditions, hence we expect the solution space to be parametrized by the vevs of any three of the scalars, with the other vevs being continuous, in general non-vanishing, functions of these three.

If one is looking for a vev for a $\mathrm{B}-\mathrm{L} \neq 0$ gauge invariant quartic scalar operator, as an induced potential term that will generate nonzero B-L so the resulting BAU is not erased by sphaleron effects, then those formed from standard model supermultiplets have been listed by Morgan [38. Those with nonzero B-L all involve Higgs vevs, except for the operator we used in our example in the previous section, so we must use it again in this case. (We should be careful here as there may be new operators that can be induced since a singlet right handed neutrino has a vev; we leave consideration of these as an exercise for the reader). In the case of our single generation flat direction (pure $16_{1}$ ) the vev arises when all the external fields lie in the $16_{1}$. In this basis the Yukawas are not generation diagonal, and the net effect is that in the diagram inducing the operator with all $166_{1}$ external legs, instead of factors of the top and bottom Yukawas, we get two factors of Yukawas whose magnitude is essentially the geometric mean of the first and second generation Yukawas; ie we have traded the coupling factors "down" in generation. In our induced operator, and hence in the final BAU produced, this results in a suppression of four or five orders of magnitude, which when compounded with the fact that our induced operator now appears at four loop order implies that even with $N$ masses raised toward the unification scale, this mechanism of baryogenesis would be, in this ansatze, of questionable viability.

While we have concentrated in this section on SO(10) inspired ansatze of the HRR type, similar issues will arise in other models incorporating a neutrino mass see-saw, such as the $\mathrm{SU}(5) \mathrm{xU}(1)$ models 46]. One should also bear in mind that the absence of B-L violation in gauge induced operators in many supersymmetric GUTs, means superpotential interactions such as neutrino masses may be the only way to produce a B-L from flat direction oscillations in many supersymmetric GUT 
models, and hence the only way to produce a BAU not subject to sphaleron erasure. As such, this possibility merits careful consideration in all prospective unified models.

\section{Conclusions}

The recent COBE observations, as well as giving us information about the inflationary epoch, define for us the cosmological parameters within which post-inflationary scenarios of baryogenesis must operate. For general classes of models, if one eschews fine tuning of parameters, we showed that the COBE observations indicate an inflaton mass of order $10^{11} \mathrm{GeV}$ and suggests a reheat temperature of order $10^{8} \mathrm{GeV}$. These low values are constraining on models of baryogenesis; in particular conventional GUT out-of-equilibrium decay scenarios have difficulty reconciling this with the observed stability of the proton. In view of this, we examined alternative mechanisms for baryogenesis, where a lepton asymmetry is created before the electroweak phase transition, and then partially reprocessed into baryons by nonperturbative electroweak effects. In both of these scenarios the lepton violating interaction generating the lepton asymmetry is the Majorana mass of a righthanded see-saw (s)neutrino. In the first mechanism, proposed by Fukugita and Yanagida, the lepton asymmetry is generated by the out of equilibrium decay of the right-handed neutrino. We have calculated the generation of lepton and baryon asymmetries via this mechanism in supersymmetric models, with sneutrino as well as neutrino decay, and shown that it proceeds unsuppressed, as in the non-supersymmetric case. We have also discussed another mechanism which we recently proposed 25 for supersymmetric models with see-saw neutrino masses, in which the Majorana masses induce effective lepton-number-violating potential terms, which act on condensate oscillations of squarks and sleptons along "flat" directions of the scalar potential. Although this mechanism only operates in supersymmetric models, it does have the advantage of being operative even in the case where the right handed (s)neutrinos are too massive to be produced after inflation. Finally, we examined the implementation of these mechanisms in the context of recently proposed ansatze for neutrino masses. The Fukugita-Yanagida mechanism was, in principle, amply able to generate a sufficient baryon asymmetry, though the actual result could not be definitely predicted; this was because phases entered which could not be measured at low energies. On the other hand, with this particular ansatze for neutrino masses, the mechanism proposed in CDO would not produce a sufficient BAU 
if one simultaneously demanded that one resolve the solar neutrino problem by MSW oscillation; this was because the MSW fit in this ansatze gave too low a Majorana mass to the right-handed neutrino. In models (and fits) with right-handed neutrino masses closer to the unification scale, this mechanism would be more efficient, and could function well (although not in the $\mathrm{SO}(10)$ models we have considered) when right-handed $N \mathrm{~s}$ are too heavy to be directly produced in inflaton decay, as required by the Fukugita-Yanagida mechanism. In conclusion, these may be efficient mechanisms for baryogenesis, which we naturally expect to arise in models incorporating realistic see-saw masses for neutrinos, and represent a viable option for cosmic baryogenesis.

\section{Acknowledgements}

We thank M.-K. Gaillard, Marcus Luty, Lawrence Hall and Mark Srednicki for very helpful discussions. The work of BAC and SD was supported in part by the Natural Sciences and Engineering Research council of Canada. The work of KAO was supported in part by DOE grant DE-AC0283ER-40105, and by a Presidential Young Investigator Award. BAC and KAO would like to thank the CERN Theory Division for kind hospitality during part of this research. 


\section{Appendix}

In this appendix, we wish to discuss the calculation of the CP-violating parameter $\epsilon$ in more detail. Since flipping a small number of signs would make $\epsilon$ zero, one must be sure that the loop diagrams of figure 2 do not correspond to an F-term vertex, because non-renormalisation theorems say that only D-terms can have loop corrections. To distinguish scalars from fermions in this section, the scalar component fields will have tildes: $\mathbf{N}=\tilde{N}+\theta N+\theta \theta F_{N}$.

In a flavour basis for the $\mathbf{L}^{i}$, there will clearly be no gauge particle contributions to $\mathrm{CP}$ violation at one loop, so one only needs to worry about diagrams involving the components of the $\mathbf{N}^{j}, \mathbf{H}$ and $\mathbf{L}^{i}$ superfields. We neglect supersymmetry-breaking and Higgs mixing masses, on the assumption that these are small compared to $M_{i}$, so $\mathbf{L}^{i}$ and $\mathbf{H}$ only have D-term propagators: if there are no mass terms $m_{i} \mathbf{L}^{i} \mathbf{L}^{i}, \mu \mathbf{H H}$ in the superpotential, then only the $<\mathbf{L} \mathbf{L}^{\dagger}>$ and $<\mathbf{H} \mathbf{H}^{\dagger}>$ two-point functions will appear. This of course is not the case for the $\mathbf{N}$ superfield, which has both $\mathrm{F}$-term $(<\mathbf{N N}>)$ and D-term $\left(<\mathbf{N N}^{\dagger}>\right)$ propagators. However, since the component diagrams of figure 2 have mass insertions on the $\tilde{N}$ and $N$ lines, one would expect $<\mathbf{N}^{i} \mathbf{N}^{i}>\sim M_{i}^{*} \Delta_{F}$ to appear. This is in fact the case, as one can see from figure 4 , the supergraph corresponding to the tree $\times$ loop $^{\dagger}$ matrix elements used to compute $\epsilon$. As previously claimed, the loop is a correction to the D-term $\mathrm{NH}^{\dagger} \mathbf{L}^{\dagger}$, so is not subject to the non-renormalisation theorems.

To calculate the lepton asymmetry produced in the decay of the $\tilde{N}^{i}$ and $N^{i}$, one only needs the $\mathrm{CP}$ violating difference $\epsilon$, which is proportional to the imaginary part of the coupling constant combination times the imaginary part of the matrix element squared. The only kinematically allowed way to have an on-shell intermediate state particle (neccesary for the [matrix element] ${ }^{2}$ to develop an imaginary part) is to put the $\mathbf{H}$ and the $\mathbf{L}$ in the loop on-shell, and the $\mathbf{N}$ off-shell. It is then easy to compute $\epsilon$ for each decay. The combination of coupling constants that needs to have

a phase, for a lepton asymmetry to be produced in the decay of $\tilde{N}^{i}$ and $N^{i}$, can be read from the supergraph to be

$$
\sum_{j} M_{i}^{*}\left(\lambda \lambda^{\dagger}\right)_{i j} M_{j}\left(\lambda^{*} \lambda^{T}\right)_{j i}
$$

At first sight the complex-conjugates on the masses appear backwards, and not terribly important in any case because one can always work in a basis where the masses are real. However, since 
propagators appear in amplitudes, and inverse propagators in the Lagrangian. $M_{i}^{*} M_{j}$ is correct. One can also check that (A1) is invariant under phase rotations of the $\mathbf{N}^{i}$, which is good, because a physical quantity $(\epsilon)$ should not depend on phase choices. It is also of some interest to get the form of (A1) in an arbitrary basis for the $\mathbf{N}^{i}$, because most models do not predict a real diagonal majorana mass matrix, and the algebra neccessary to check if there is a phase is simplified if one does not have to rotate to the basis where $M_{i} \in \mathcal{R}$. If $\hat{M}$ is a symmetric majorana mass matrix, and $\hat{\lambda}$ is the yukawa coupling in this basis, then $\hat{M}$ can be diagonalized in this basis by a unitary matrix $U$ :

$$
U^{T} \hat{M} U=\operatorname{diag}\left[M_{1}, M_{2}, M_{3}\right]
$$

The coupling constant combination that needs a phase is therefore

$$
\left(U^{\dagger} \hat{M}^{\dagger} \hat{\lambda} \hat{\lambda}^{\dagger} \hat{M} \hat{\lambda}^{*} \hat{\lambda}^{T} U\right)_{i i}
$$

Note that this is true in any basis, provided that $U$ diagonalizes the majorana mass matrix. 


\section{References}

[1] A.D. Sakharov, JETP Lett. 5(1967)24.

[2] for reviews see: E.W. Kolb and M.S. Turner, Ann. Rev. Nucl. Part. Sci.33(1983)645;

A. Dolgov, Yukawa Institute preprint YITP/K-940, 1991.

[3] for reviews see: A.D. Linde, Particle Physics And Inflationary Cosmology Harwood (1990);

K.A. Olive, Phys.Rep. C190(1990)307:

[4] V. Kuzmin, V. Rubakov, and M. Shaposhnikov, Phys. Lett. B155(1985)36.

[5] P. Arnold and L. McLerran, Phys. Rev. D36(1987)581; D37(1988)1020.

[6] M. Fukugita and T. Yanagida, Phys. Rev. D42(1990)1285.

[7] J. Harvey and M. Turner, Phys. Rev. D42(1990)3344.

[8] A. Nelson and S. Barr, Phys. Lett. B246(1990)141.

[9] B.A. Campbell, S. Davidson, J. Ellis, and K.A. Olive, Phys. Lett. B256(1991)457; Astroparticle Physics 1 (1992) 77; CERN-TH.6642/92(1992).

[10] W. Fischler, G.F. Giudice, R.G. Leigh, and S. Paban, Phys. Lett. B258(1991)45.

[11] L.E. Ibáñez and F. Quevedo, CERN-TH.6433/92(1992).

[12] H. Dreiner and G. Ross, OUTP-92-08P(1992).

[13] M.E. Shaposhnikov, Nucl. Phys. B287(1987)757; B299(1988)797;

A.I. Bochkarev, V. Kuzmin, and M.E. Shaposhnikov, Phys. Lett. B244(1990)275;

A.I. Bochkarev,S.Yu. Khlebnikov, and M.E. Shaposhnikov, Nucl. Phys. B329(1990)490.

[14] L. McLerran, Phys. Rev. Lett. 62(1989)1075;

N. Turok and J. Zadrozny, Phys. Rev. Lett. 65(1990)2331; Nucl. Phys. B358(1991)471;

L. McLerran, M.E. Shaposhnikov, N. Turok and M. Voloshin Phys. Lett. B256(1991)251; 
A. Cohen, D. Kaplan and A. Nelson, Nucl. Phys. B349(1991)727; Phys. Lett. B263(1991)86; G.W. Anderson and L.J. Hall, Phys. Rev. D45(1992)2685.

[15] M. Dine, P. Huet, R.S. Singleton Jr. and L. Susskind, Phys. Lett. B257(1991)351;

M. Dine, P. Huet,and R.S. Singleton Jr., Nucl. Phys. B375(1992)625;

M. Dine, R.G. Leigh, P. Huet, A. Linde and D. Linde, Slac preprints 5740 and 5741 (1992).

[16] M. Carrington, Phys. Rev. D45(1992)2933.

[17] For a review see: D. Karlen in: Proceedings of the Conference Beyond The Standard Model III, Ottawa, Canada (1992)

[18] G.F. Giudice,Phys. Rev. D45(1992)3177;

S. Myint, BU preprint HEP-92-4.

[19] M. Pietroni, Padova preprint DFPD/92/TH/36.

[20] S. Weinberg, Phys. Rev. Lett. 42(1979)850.

[21] D. Toussaint, S.B. Treiman, F. Wilczek, and A. Zee, Phys. Rev. D19(1979)1036

[22] I. Affleck and M. Dine, Nucl. Phys. B249(1985)361.

[23] M. Fukugita and T. Yanagida, Phys. Lett.B174(1986)45.

[24] M. Luty, Phys. Rev. D45(1992)455.

[25] B.A. Campbell, S. Davidson, and K.A. Olive, Alberta-Thy-31-92/ CfPA-92-25/ UMNTH-111392, September 1992.

[26] F.L. Wright et al. Ap. J. Lett. 396(1992)L13.

[27] A. Albrecht and R. Brandenberger, Phys. Rev. D31(1985)1225.

[28] A.D. Linde, Phys. Lett. B129(1983)177. 
[29] P. Binetruy and M.K. Gaillard, Phys. Rev. D34(1986)3069.

K. Freese, J.A. Frieman, and A.V. Olinto, Phys. Rev. Lett. D65(1990)3233.

F.C. Adams et al. FERMILAB-Pub-92/202-A (1992).

[30] D.V. Nanopoulos, K.A. Olive, M. Srednicki, and K. Tamvakis, Phys. Lett. B123(1983)41;

R. Holman, P. Ramond, and G.G. Ross, Phys. Lett. B137(1984)343.

[31] J. Ellis, K.Enquist, D.V. Nanopoulos, K.A. Olive, M. Srednicki, Phys. Lett. B152(1985)175.

[32] S. Hawking, Phys. Lett. B115 (1982)295;

A.A. Starobinsky, Phys. Lett. B117 (1982)175;

A. Guth and S.-Y. Pi, Phys. Rev. Lett. 49(1982) 1110;

J. Bardeen, P. Steinhardt and M. Turner, Phys. Rev. D28(1983)679;

D.H. Lyth, Phys. Rev. D31(1985)1792.

[33] P.J.E. Peebles, Ap.J. Lett. 263(1982) L1;

L.F. Abbott and M.B. Wise, Phys. Lett. B135(1984) 279;

[34] R.L.Davis, H.M. Hodges, G.F. Smoot, P.J. Steinhardt, and M.S. Turner, Fermilab-PUB92/168(1992);

A.R. Liddle and D.H. Lyth, Sussex AST 92/6-1(1992);

D. Salopek, DAMTP/R-92/26 (1992).

[35] J. Ellis, K. Enqvist, D.V. Nanopoulos, and K.A. Olive, Phys. Lett. B191(1987) 343.

[36] A.D. Linde, Phys. Lett. B160(1985)243.

[37] B.A. Campbell, J. Ellis, D.V. Nanopoulos, and K.A. Olive, Mod. Phys. Lett. A1(1986)389.

[38] D. Morgan, Nucl. Phys. B364(1991)401.

[39] R. Barbieri, D.V. Nanopoulos and A. Masiero, Phys. Lett. B98(1981)191;

A. Masiero, D.V. Nanopoulos, K. Tamvakis and T. Yanagida, Z. Phys. C17(1983)33.

[40] J. Harvey, P. Ramond, and D. Reiss, Phys. Lett. B92(1980) 309, Nucl. Phys. B199(1980)223. 
[41] S. Dimopoulos, L. Hall and S. Raby, Phys. Rev. Lett. 68 (1992) 1984; Phys. Rev D45(1992) 4192; LBL-32484 (1992).

[42] H. Fritzsch, Phys. Lett. B70(1977)437.

[43] H. Georgi and C. Jarlskog, Phys. Lett. B86(1979)97.

[44] M. Gell-Mann, P. Ramond, and R. Slansky, in Supergravity, eds. D.Z. Freedman and P. van Nieuwenhuizen, North Holland (1979);

T. Yanagida, in Proceedings of the Workshop on the Unified Theory and The Baryon Number of the Universe, eds O. Sawada and S. Sugamoto. KEK79-18 (1979).

[45] M. Fukugita and T. Yanagida, Mod. Phys. Lett. A6(1991)645.

[46] J. Ellis, D.V. Nanopoulos, and K.A. Olive, Cern preprint TH.6721/92 and Minnesota preprint UMN-TH-1117/92. 


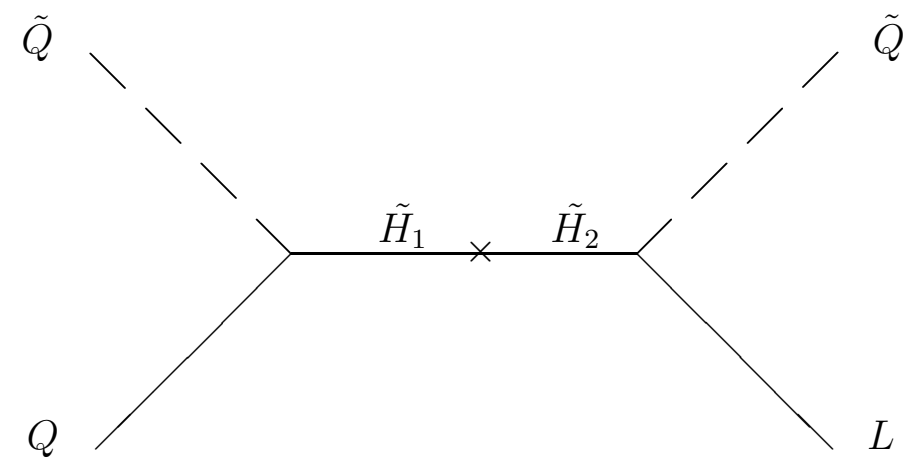

Figure 1: Diagram inducing baryon number violating dimension five operator in susy SU(5) 
a)

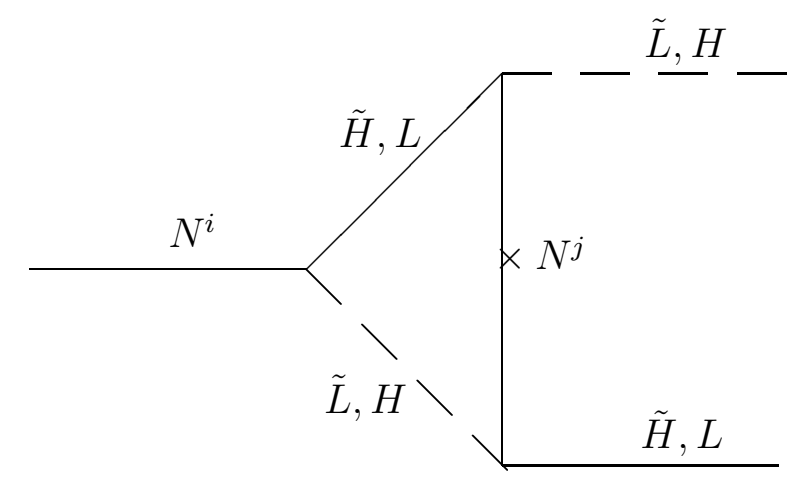

b)
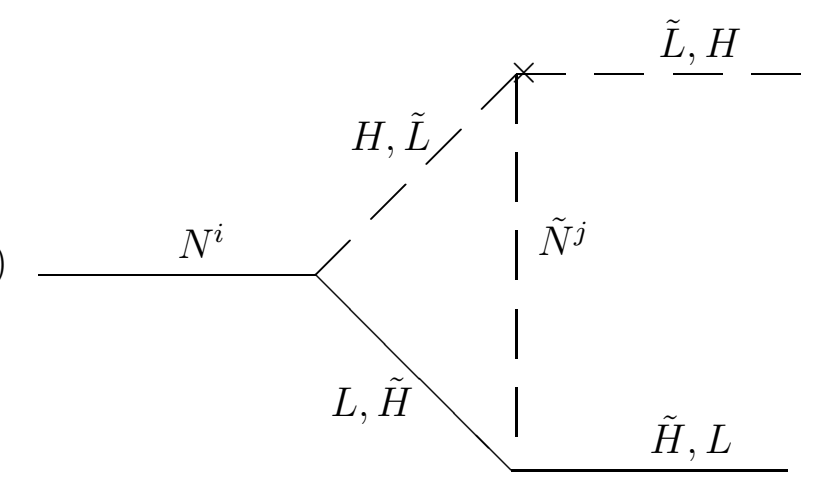

c)

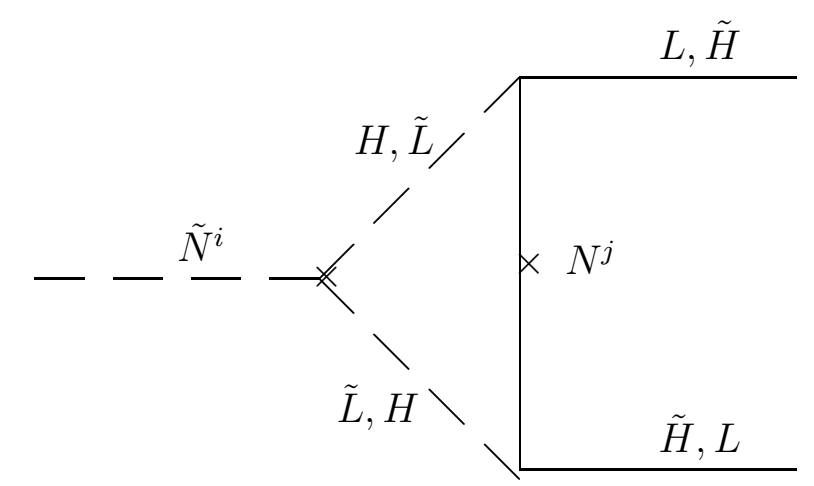

d)

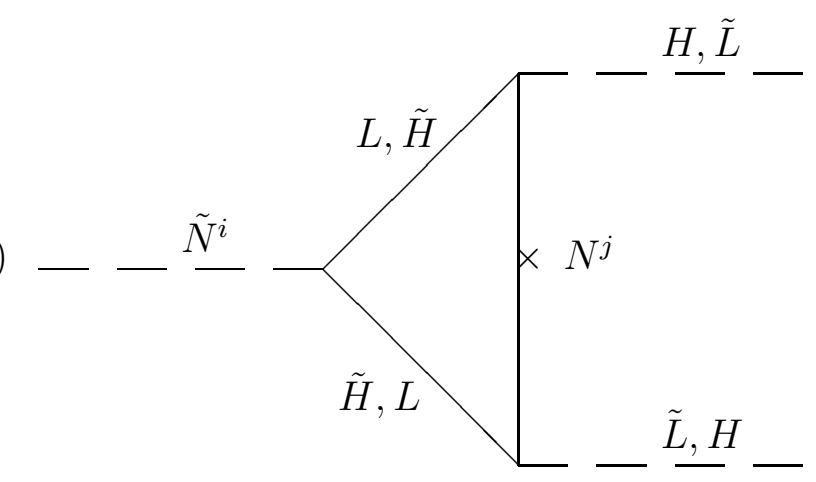

Figure 2: One-loop diagrams contributing to $\mathrm{CP}$ violation in neutrino and sneutrino decays; only diagram a) with $H$ and $L$ will contribute in the non-supersymmetric case 
Figure 3: Diagram inducing lepton number violating potential terms from the neutrino mass see-saw superpotential after supersymmetry breaking. 


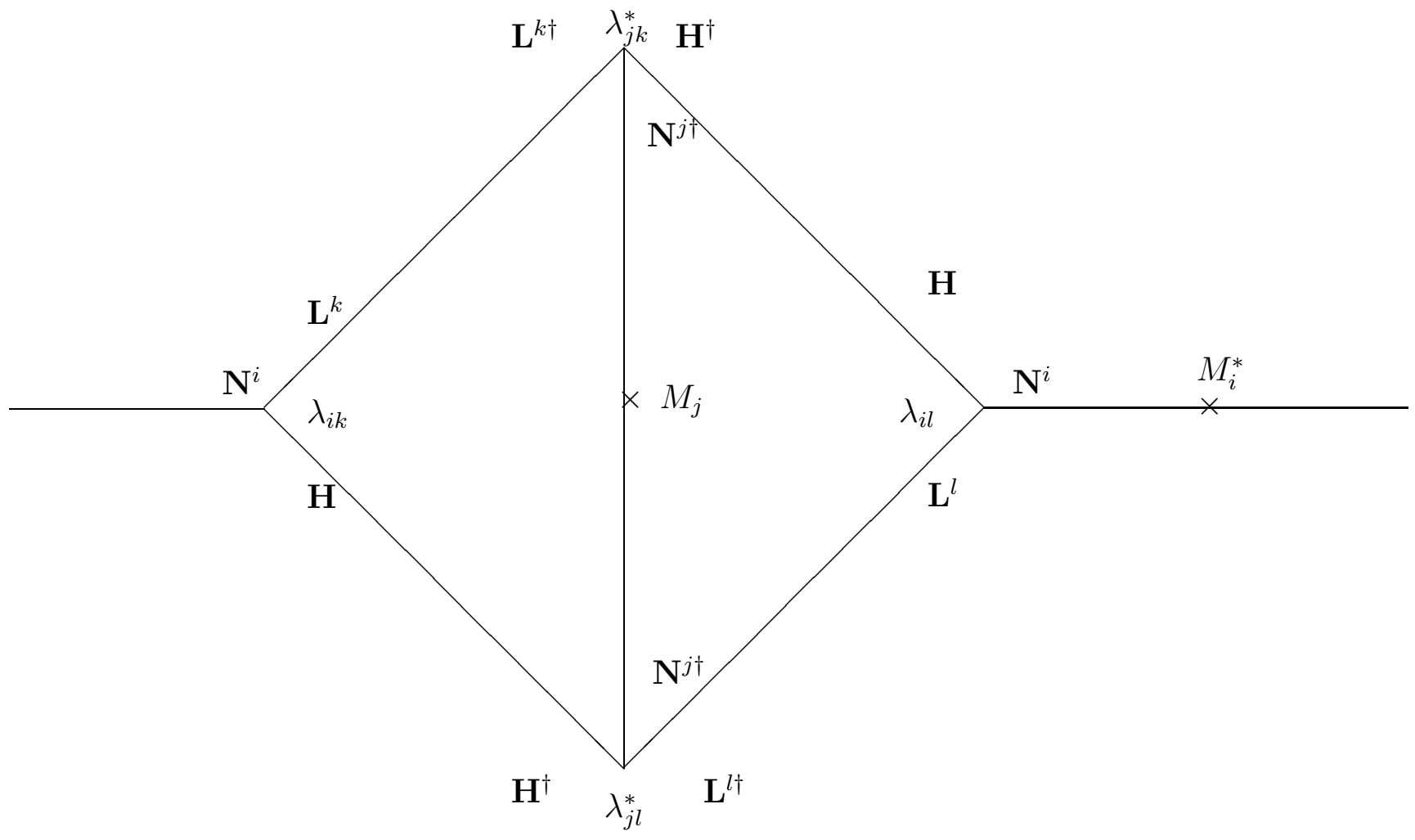

Figure 4: supergraph corresponding to the loop $\times$ tree $^{\dagger}$ matrix elements providing $\mathrm{CP}$ violation in heavy singlet neutrino and sneutrino decays 